Widerständen horizontalen Verlauf der $q$-Kurven zu schließen, daß die Abweichungen von (3b) erst bei höheren Widerständen merklich werden.

Die geradlinige Extrapolation der Halbwertszeitkurven auf $R=\infty$ führt nicht für alle Stellungen des magnetischen Shunts zum gleichen Wert der verbleibenden Halbwertszeit, was darauf schließen läßt, daß der größte Teil der restlichen Dämpfung Rahmendämpfung und nicht Luftdämpfung ist (die niederohmige Spule des Galvanometers war bei den Messungen offen).

Für konstante, genügend kleine Widerstände $R_{\mathrm{a}}$ entnimmt man der Abb. 1 die durch Gl. (8) geforderte Proportionalität der Halbwertszeiten mit dem Quadrat der durch den magnetischen Nebenschluß veränderten Größen $q$.

Diagramme nach Art der Abb. 1 sind also geeignet, über den Widerstands- und Empfindlichkeitsbereich Aufschluß zu geben, in dem ein Galvanometer als $\mathrm{KG}$ arbeiten kann.

Die Veranlassung zu den hier dargestellten Überlegungen und Messungen war die Verwendung von KG für zahlreiche verschiedene Arbeiten der magnetischen Abteilung von Prof. W. Ge r la ch s Institut an der Universität München. Der Verf. würde sich sehr freuen, wenn sein verehrter Lehrer bei der Planung und Ausführung weiterer, hoffentlich noch recht zahlreicher magnetischer Arbeiten aus den hier abgeleiteten Diagrammen einen kleinen Nutzen ziehen könnte.

\title{
Polarisationserscheinungen bei der Beugung am Spalt"
}

\author{
Von Willy Hartnagel und Eugen Kappler \\ Aus dem Physikalischen Institut der Universität München \\ (Z. Naturforschg. 4 a, 498-506 [1949]; eingegangen am 15. April 1949)
}

\begin{abstract}
Es werden einige neue Beobachtungen über die Polarisation bei der Beugung am Einzelspalt mitgeteilt, auf Grund derer eine einfache Theorie der Beugung am Spalt entwickelt wird. Unsere Vorstellung ist die, daß das durch den Spalt hindurchgehende Licht aus zwei Anteilen besteht, wovon der erste, bei weiten Spalten weitaus überwiegende Anteil aus den zwischen den Spaltbacken liegenden Teilen des Spaltes kommt und identisch ist mit der aus der einfachen Kirchhoffschen Beugungstheorie sich ergebenden Intensitätsverteilung. Der Polarisationszustand dieses Anteils ist derselbe wie derjenige der einfallenden Welle. Der zweite Anteil geht von den Spaltbacken aus; er besitzt eine Phasenverschiebung gegenüber dem Kirchhoffschen Anteil, die im allgemeinen für die $\pi$ - und $\sigma$-Komponente verschieden sein wird. Seine Intensität und Phase hängen sehr wesentlich von der Form der Spaltbacken und den optischen Konstanten des Spaltmaterials ab.
\end{abstract}

$\mathrm{P}$ olarisationseffekte bei der Beugung sind besonders in der älteren Literatur vielfach Gegenstand von experimentellen und theoretischen Untersuchungen gewesen. Eingehender untersucht ist im wesentlichen die Beugung am Gitter. Die Ergebnisse dieser Untersuchungen sind z. Tl. widersprechend.

Die Polarisation bei der Beugung am Einzelspalt ist nicht so eingehend studiert worden. Im folgenden werden einige neue Beobachtungen mitgeteilt ${ }^{1}$. Sie sind bedingt durch die physikalischen

* Hrn. Prof. Dr. Walther Gerlach zum 60. Geburtstag gewidmet.

1 Ǔber einen Teil dieser Beobachtungen hat E. K a p pl e r, Physik. Blätter 3, 163 [1947], kurz berichtet. Außerdem wurde auf der Tagung der Physikalischen Gesellschaft Württemberg-Baden in Heidenheim am 15. November 1947 darüber vorgetragen (s. Physik. Blätter 3, 403 [1947]).
Vorgänge an den Spalträndern, für welche die Form der Spaltbacken und die optischen Konstanten des Spaltmaterials maßgebend sind, Vorgänge, die in den theoretischen Untersuchungen im Sinne der Sommerfeldschen Methode in dieser Allgemeinheit nicht behandelt worden sind. Im zweiten Teil wird eine einfache Theorie gegeben, die nicht den Anspruch auf eine quantitative Beschreibung der sehr verwickelten Verhältnisse erhebt, in der aber, wie uns scheinen will, die physikalisch hauptsächlich wirksamen Faktoren zum Ausdruck kommen.

\section{Experimenteller Teil}

\section{A. Versuchsanordnung}

Es wurde die Beugungsfigur des Spaltes in Fraunhoferscher Anordnung beobachtet bzw. photographiert. 
Abb. 1 zeigt den Strahlengang. Bei den in den Abb. 3 und 4 wiedergegebenen Aufnahmen diente als Lichtquelle eine Hg-Höchstdrucklampe HBO 501 mit Filtern, und zwar bei den Aufnahmen der Abb. 3 ein Schott-Filter OG 4, welches im wesentlichen die grüne, die gelbe und die schwächeren roten $\mathrm{Hg}$-Linien durchläßt; bei den Aufnahmen der Abb. 4 wurde ein Wrattenfilter verwendet, das nur für die gelbe und die roten $\mathrm{Hg}$-Linien durchlässig ist. Die Aufnahmen der Abb. 5 wurden mit dem Licht einer Na-Dampflampe hergestellt, das die beiden Schott-Filter OG 2 und OG 3 passierte, so daß nur die D-Linien wirksam waren. Sämtliche Aufnahmen wurden mit der im Gelb sehr empfindlichen Peromnia-Platte von Perutz hergestellt.

Zur Ermittlung der Intensitätsverteilung wurde die mit einem Zeißschen Spektrallinienphotometer
B. B e obachtungsergebnisse

1. Befindet sich der Spalt zwischen gekreuzten Polarisatoren, so erfolgt im allgemeinen keine vollständige Auslöschung. Zwar wird der weitaus größte Teil des durch den Spalt hindurchgehenden Lichtes ausgelöscht, aber ein kleiner Teil des auffallenden Lichtes geht noch durch den Analysator hindurch, und man beobachtet ein neues Beugungsspektrum, das von dem gewöhnlichen, etwa mit unpolarisiertem Licht erhaltenen, verschieden ist. Die Erscheinung ist am ausgeprägtesten bei einem Polarisationsazimut von $45^{\circ}$ gegen die Spaltbacken, während die Auslöschung eine vollständige ist, wenn die Polarisatoren

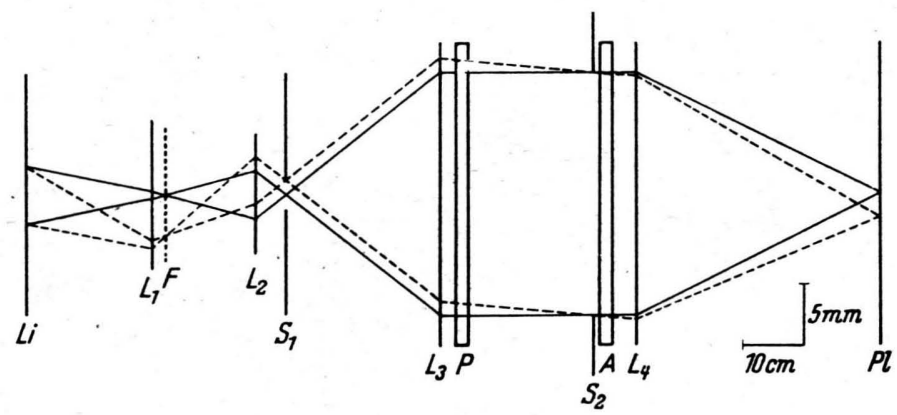

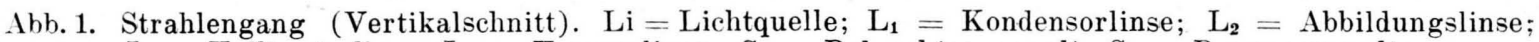
$\mathrm{L}_{3}=$ Kollimatorlinse; $\mathrm{L}_{4}=$ Kameralinse; $\mathrm{S}_{1}=$ Beleuchtungsspalt; $\mathrm{S}_{2}=$ Beugungsspalt; $\mathrm{P}=$ Polarisator; $\mathrm{A}=$ Analysator $\mathrm{F}=$ Stufenfilter $; \mathrm{Pl}=$ Photographische Platte.

photometrierte Beugungsfigur auf Intensitäten umgerechnet mit Hilfe von Intensitätsmarken. Letztere wurden mit Hilfe eines Stufenfilters hergestellt, dessen Durchlässigkeiten für die D-Linien ermittelt worden waren, und das so in den Strahlengang eingeschwenkt werden konnte, daß es durch die Linse $\mathrm{L}_{2}$ auf den Beleuchtungsspalt des Kollimators abgebildet wurde.

Zum Studium der Polarisationsverhältnisse der unter B 1 beschriebenen Beobachtung befand sich der Spalt zwischen den Glan-Thompson-Prismen P und A. Bei den unter B 2 mitgeteilten Beobachtungen wurde der Polarisator $\mathrm{P}$ entfernt, und an der Stelle des Analysators A befand sich ein Wollaston-Prisma, dessen Hauptschwingungsrichtungen parallel bzw. senkrecht zu den Spaltbacken gerichtet waren ( $\pi$ und $\sigma$ ).

Das von der Natriumdampflampe ausgehende Licht war nicht vollständig unpolarisiert [infolge der verschiedenen Durchlässigkeit der vertikal stehenden zylindrischen Entladungsröhre für die vertikal $(\pi)$ und horizontal $(\sigma)$ schwingende Komponente]. Die senkrecht zum Spalt schwingende $\sigma$-Komponente war daher um etwa $4 \%$ stärker als die parallel zum Spalt schwingende $\pi$-Komponente. Dies bedingt bei den in Abb. 6 gezeigten Photometerkurven eine geringfügige Korrektion im Sinne einer Verminderung der Intensität der $\sigma$-Komponente gegenüber der $\pi$-Komponente. parallel bzw. senkrecht $z u$ den Spaltbacken stehen. Diese neue Beugungserscheinung hat folgende Eigenschaften (s. die Abb. 3 I b, 3 II b, 4 b und $4 \mathrm{~d}^{2}$ ): Zum Unterschied von der gewöhnlịchen Beugungsfigur des Spaltes haben alle Maxima und alle Minima denselben Abstand voneinander. Dabei liegen die Maxima ungefähr an den Stellen, wo bei der gewöhnlichen Beugungsfigur die Minima liegen, und umgekehrt. Die Erscheinung ist im allgemeinen sehr lichtschwach; sie kann mit dem Licht einer Bogenlampe gerade noch gut wahrgenommen werden, dagegen nicht mehr z. B. mit der Na-Dampf-Lampe.

Die Aufnahmen der Abb. 3 beziehen sich auf zwei verschiedene Stahlspalte von ungefähr gleicher Spaltweite, aber von verschiedener Form der Spaltbacken, und zwar die Aufnahmen $3 \mathrm{I}$ auf einen aus Rasierklingen hergestellten Spalt mit Spaltbacken von sehr kleinem Krümmungsradius (Spalt I), die Aufnahmen 3 II auf einen solchen mit wesentlich größerem Krümmungsradius (Spalt II)

2 Vgl. 'Tafel, S. 504 a. 
(vgl. Abb.2). Beide Abbildungen enthalten zum Vergleich die mit unpolarisiertem Licht sich ergebende Beugungsfigur der beiden Spalte (Aufnahme $3 \mathrm{Ia}$ und $3 \mathrm{II}$ a). Um Überstrahlungen durch die intensive nullte Ordnung zu verhindern, wurde diese mit einer Papierblende in der Ebene der photographischen Platte ausgeblendet. In beiden Fällen ist die neue Beugungsfigur ähnlich derjenigen eines Doppelspaltes, bei dem der Spaltabstand gleich der Breite des verwendeten Spaltes wäre. Bei beiden Spalten nimmt die Intensität in den Maxima mit zunehmender Ordnungszahl ab, und zwar rascher beim Rasierklingenspalt. Wesentliche Unterschiede zeigen sich in der Intensität der Erscheinung. Sie ist beim Rasier-

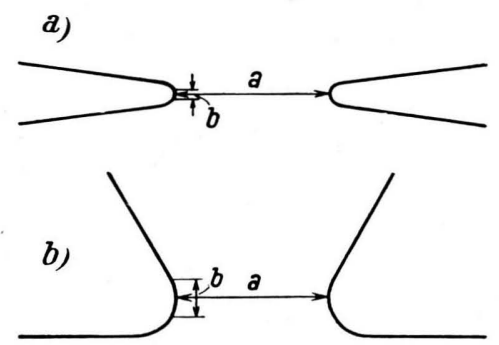

Abb. 2. Form der Spaltbacken. a) Spalt I (Rasierklingenspalt), b) Spalt II.

klingenspalt wesentlich schwächer als beim Spalt II (vgl. die Belichtungszeiten der Abb. $3 \mathrm{Ib}$ und 3 II b). Zum Unterschied von reinen Doppelspaltinterferenzen ist die Intensität in den Minima nicht gleich Null, sondern besitzt einen endlichen Wert, der mit wachsender Ordnungszahl zunimmt und bei dem Spalt II sehr viel stärker ist als bei dem Spalt I. Diese Intensität in den Minima ist nicht etwa vorgetäuscht durch die Inhomogenität des mit dem OG 4-Filter gefilterten Hg-Lichtes oder der endlichen Breite des Beleuchtungsspaltes, der bei der Abb.3, im Interesse kürzerer Belichtungszeiten, verhältnismäßịg weit gewählt worden war $(800 \mu)$. Dies beweist Abb. 4, welche Aufnahmen enthält, die im wesentlichen nur mit der gelben $\mathrm{Hg}$-Linie aufgenommen worden sind, und wo die Spaltbreite des Beleuchtungsspaltes nur $200 \mu$ betrug. Die Aufnahmen a, b und c, d beziehen sich auf zwei verschiedene Spaltbreiten.

2. Die Intensitätsverteilung im Beugungsspektrum ist verschieden für die parallel und senkrecht zum Spalt liegende Polarisationsrichtung, und beide sind verschieden von der aus der Kirchhoffschen Theorie folgenden Intensitätsverteilıng $\left(\sin ^{2} \varphi / \varphi^{2}\right) . \mathrm{Zu}$ ihrer Ermittlung fällt unpolarisiertes Licht auf den Spalt. Das durch den Spalt hindurchgehende Licht durchläuft ein vor der Kameralinse angebrachtes Doppelbildprisma, dessen Hauptschwingungsrichtungen parallel und senkrecht zu den Spaltbacken liegen. Die Abb. 5 zeigt Aufnahmen mit Spalt II für drei verschiedene Spaltbreiten, und zwar bei der Aufnahme a) etwa $44 \mu$, bei b) etwa $18 \mu$, bei c) etwa $11 \mu$ und eine Aufnahme (d) mit Spalt I (Spaltbreite etwa $16,5 \mu)$. Die obere Aufnahme gilt jeweils für die $\pi$-Komponente, die untere für die $\sigma$-Komponente. Sämtliche Aufnahmen wurden mit den D-Linien hergestellt. Die Breite des Beleuchtungsspaltes betrug bei der Aufnahme 5 a $100 \mu$ und bei den Aufnahmen $5 \mathrm{~b}$, c und d $200 \mu$. Damit war mit hinreichender Genauigkeit gewährleistet, daß (mit Ausnahme des 1. Minimums von 5a, vgl. Abb. 6a) in die Minima kein Licht infolge der endlichen Ausdehnung des Beleuchtungsspaltes gelangen konnte. In Abb. 6 sind die Photometerkurven für die eine Hälfte der Aufnahmen der Abb. 5 abgebildet. Auch bei diesen Aufnahmen wurde die nullte Ordnung ausgeblendet.

Aus diesen Aufnahmen lassen sich folgende Tatsachen ablesen:

a) Maxima und Minima liegen für die $\pi^{-}$und $\sigma$-Komponente an verschiedenen Stellen; sie sind für die $\sigma$-Komponente weiter von der nullten Ordnung entfernt als für die $\pi$-Komponente. Je enger der Spalt ist, um so ausgeprägter ist diese Erscheinung. Diese relative Verschiebung der Extremstellen ist wesentlich stärker bei Spalt II als bei Spalt I.

b) Die Intensität in den Maxima, einschließlich der nullten Ordnung, ist für die $\sigma$-Komponente geringer als für die $\pi$-Komponente. Der Intensitätsunterschied (bezogen auf die einfallende Intensität ) zwischen $\pi$ und $\sigma$ wächst ebenfalls mit abnehmender Spaltbreite.

c) Die Intensität in den Minima ist nicht gleich Null, wie die Kirchhoffsche Intensitätsverteilung verlangen würde. Sie ist für die $\pi$-Komponente größer als für die $\sigma$-Komponente. Sie nimmt für enge Spalte beträchtliche Werte an, so daß mit abnehmender Spaltbreite der Intensitätsunterschied zwischen Maxima und Minima immer kleiner wird.

Die Intensität in den Minima scheint sowohl für Spalt I als auch für Spalt II von derselben Größenordnung zu sein (zum Unterschied von der Ver- 


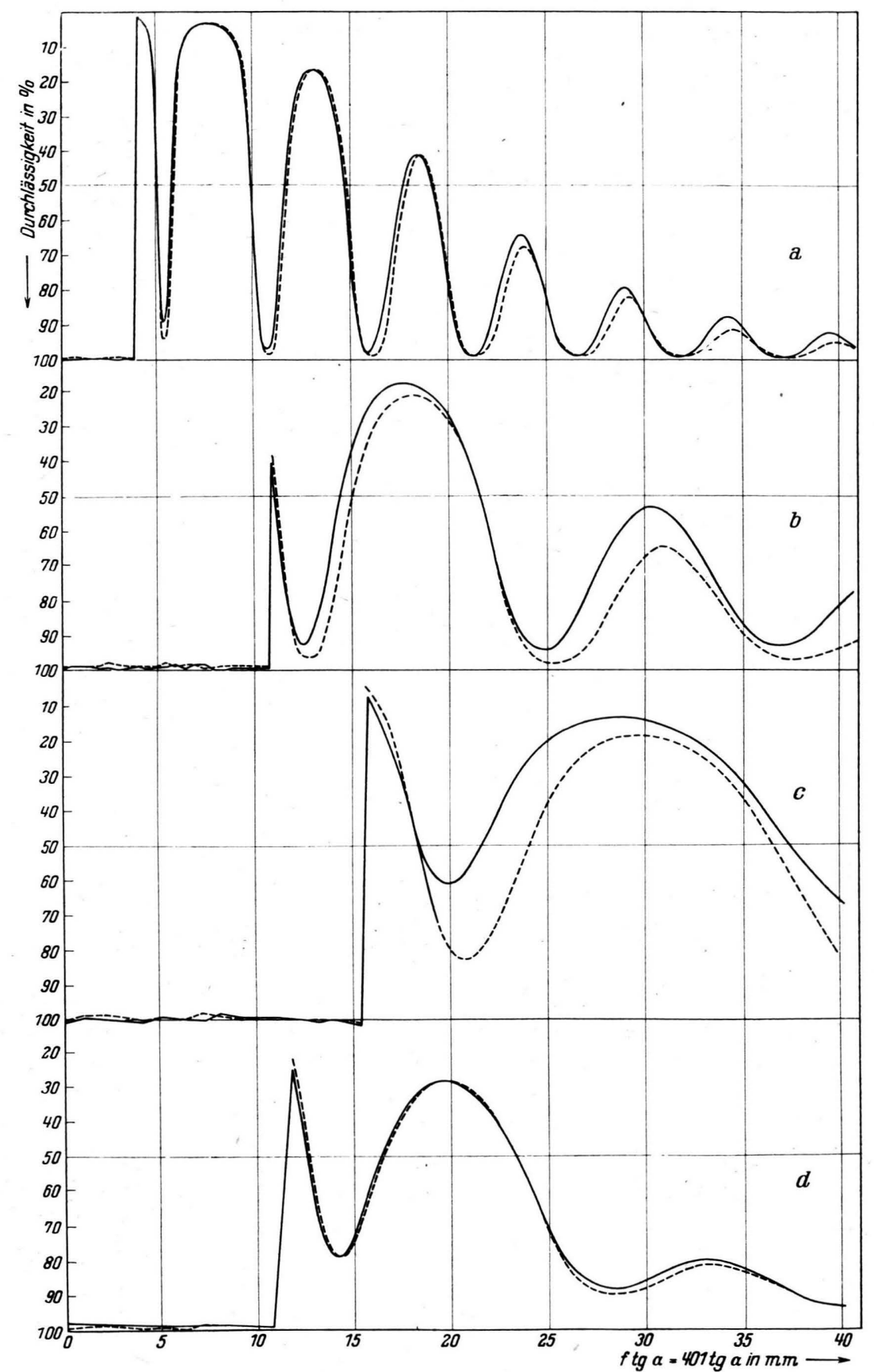

Abb. 6. Photometerkurven der Aufnahmen von Abb. 5 (vgl. die dortigen Angaben). Ausgezogen: $\pi$-Komponente, gestrichelt: $\sigma$-Komponente.

schiebung der Extremstellen). Obwohl dieser den Spalt I betreffende Befund an mehreren Spalten gefunden wurde, scheint er uns nicht ganz gesichert, da kleine Unebenheiten der Rasierklingen, - die nur selten nicht vorhanden sind, denselben Effekt bewirken könnten. d) Bei weiten Spalten schneiden sich die Intensitätskurven für $\pi$ und $\sigma$ ungefähr an den Stellen $\varphi=\pi / 2,3 \pi / 2,5 \pi / 2$ usw. $[\varphi=(\pi / \lambda) a \sin \alpha$, wobei $a=$ Spaltbreite, $\lambda=$ Wellenlänge, $\alpha=$ Ablenkungswinkel bedeuten; vgl. Abb. 6 a]. Bei engen Spalten treten keine Schnittpunkte mehr auf (Abb. 6c). 


\section{Theoretischer Teil}

Die unter I B 1 mitgeteilte Beobachtung legt die Annahme nahe, daß das durch den Spalt hindurchgehende Licht aus zwei Anteilen besteht, wovon der erste - und zwar bei weiten Spalten der weitaus größte Anteil - dieselbe Schwingungsrichtung besitzt wie das auffallende Licht und durch die Kirchhoffsche Intensitätsformel beschrieben wird. Er wird also bei gekreuzter Stellung der Polarisatoren nicht durchgelassen und kommt aus den zwischen den Spaltbacken liegenden Teilen des Spaltes. Der zweite Anteil hat seinen Ausgangspunkt in den Spaltbacken selbst (,Reflexion“ an den Spaltbacken). Er besitzt gegenüber dem einfallenden und damit auch gegenüber dem aus der Spaltmitte kommenden ersten Teil eine Phasen-

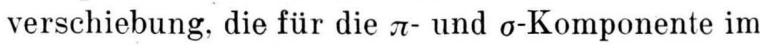
allgemeinen verschieden groß ist, so daß es sich bei diesem Anteil, z. B. bei einem Polarisationsazimut von $45^{\circ}$, um elliptisch polarisiertes Licht handelt, das bei gekreuzter Stellung der Polarisatoren den Analysator passieren kann und die unter I B 1 mitgeteilten „Doppelspaltinterferenzen" zustande bringt. Außerdem wird auch die Amplitude dieser Randwellen für die $\pi^{-}$und $\sigma^{-}$ Komponente verschieden sein, was unter allen Umständen zu den beobachteten „Doppelspaltinterferenzen" führt, auch für den Fall, daß $\pi^{2}$ und $\sigma$-Komponente keine Phasendifferenz gegeneinander besitzen.

Um zu Aussagen über die Eigenschaften dieser Randwelle zu gelangen, die zweifellos sehr verwickelt sind, werden wir zunächst von sehr primitiven Annahmen ausgehen. Durch Vergleich der aus diesen Annahmen sich ergebenden Folgerungen mit den Beobachtungen werden wir sehen, in welcher Weise diese Annahmen zu vervollkommnen sind.

\section{Annahme 1}

Die Spaltbacken stellen lineare Lichtquellen dar, d. h. die Breite $b$ der ,,reflektierenden“ Zone der Spaltbacken (vgl. Abb. 2) ist klein im Vergleich zur Wellenlänge, so daß Phasenunterschiede innerhalb dieser Zone vernachlässigt wer- den können. Die Amplitude der emittierten Randwelle sei unabhängig von der Richtung; d. h. es handle sich um zwei Zylinderwellen (vgl. Abb. 7, Kurve a). Dasselbe werde für die Phase angenommen. Wir führen also eine konstante (richtungsunabhängige) Phasendifferenz $\delta_{\pi}$ bzw. $\delta_{\sigma}$ gegenüber dem Kirchhoffschen Anteil für die $\pi$ - bzw. $\sigma$-Komponente ein.

Mit dieser Annahme ergibt sich für die Amplitude der $\pi^{-}(\sigma)$-Komponente des in beliebiger Richtung $\alpha$ abgebeugten Lichtes folgendes:

$$
\begin{aligned}
& A_{\pi(\sigma)}=\frac{a}{i 2 \varphi}\{\exp (i \varphi)-\exp (-i \varphi)\} \\
& \quad+b c_{\pi(\sigma)} \exp \left(i \delta_{\pi(\sigma)}\right)\{\exp (i \varphi+\exp (-i \varphi)\} .
\end{aligned}
$$

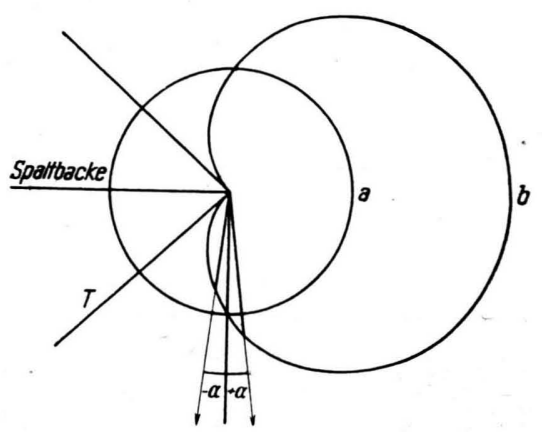

Abb. 7. Amplitudendiagramm der Randwelle. Kurve a: nach Annahme 1, Kurve b: nach Annahme 2 mit $d=1 /$.

Hierbei stellt der erste Ausdruck den Kirchhoffschen Anteil und der zweite Ausdruck die Randwelle dar. Die Amplitude des einfallenden Lichtes ist hierbei gleich 1 gesetzt. Die als richtungsunabhängig angenommene Amplitude der Randwelle ist proportional zur Ausdehnung $b$ der „,reflektierenden“ Spaltzone und wird durch $b c$ gekennzeichnet, wobei $c$ eine $\mathrm{Zahl}<1$ und ein Maß für das „Reflexionsvermögen“ der Spaltbacken ist.

Für die Intensität ergibt sich, wenn der Spalt zwischen zwei Polarisatoren sich befindet, mit dem Azimut $\beta$ für den Polarisator und dem Winkel $\gamma$ zwischen Polarisator und Analysator:

$$
I=a^{2} \frac{\sin ^{2} \varphi}{\varphi^{2}} L_{1}+4 b^{2} \cos ^{2} \varphi L_{2}+4 a b \frac{\sin \varphi \cos \varphi}{\varphi} L_{3},
$$

wobei die Buchstaben $L$ folgende Bedeutung haben: 
$L_{1}=\cos ^{2} \gamma$,

$$
\begin{aligned}
& L_{2}=c_{\pi}^{2} \cos ^{2} \beta \cos ^{2}(\beta+\gamma)+c_{\sigma}^{2} \sin ^{2} \beta \sin ^{2}(\beta+\gamma)+1 / 2 c_{\pi} c_{\sigma} \cos \left(\delta_{\pi}-\delta_{\sigma}\right) \sin 2 \beta \sin 2(\beta+\gamma), \\
& L_{3}=c_{\pi} \cos \delta_{\pi}\left\{\cos ^{2} \beta \cos ^{2}(\beta+\gamma)+1 / 4 \sin 2 \beta \sin 2(\beta+\gamma)\right\} \\
& \quad+c_{\sigma} \cos \delta_{\sigma}\left\{\sin ^{2} \beta \sin ^{2}(\beta+\gamma)+1 / 4 \sin 2 \beta \sin 2(\beta+\gamma)\right\} .
\end{aligned}
$$

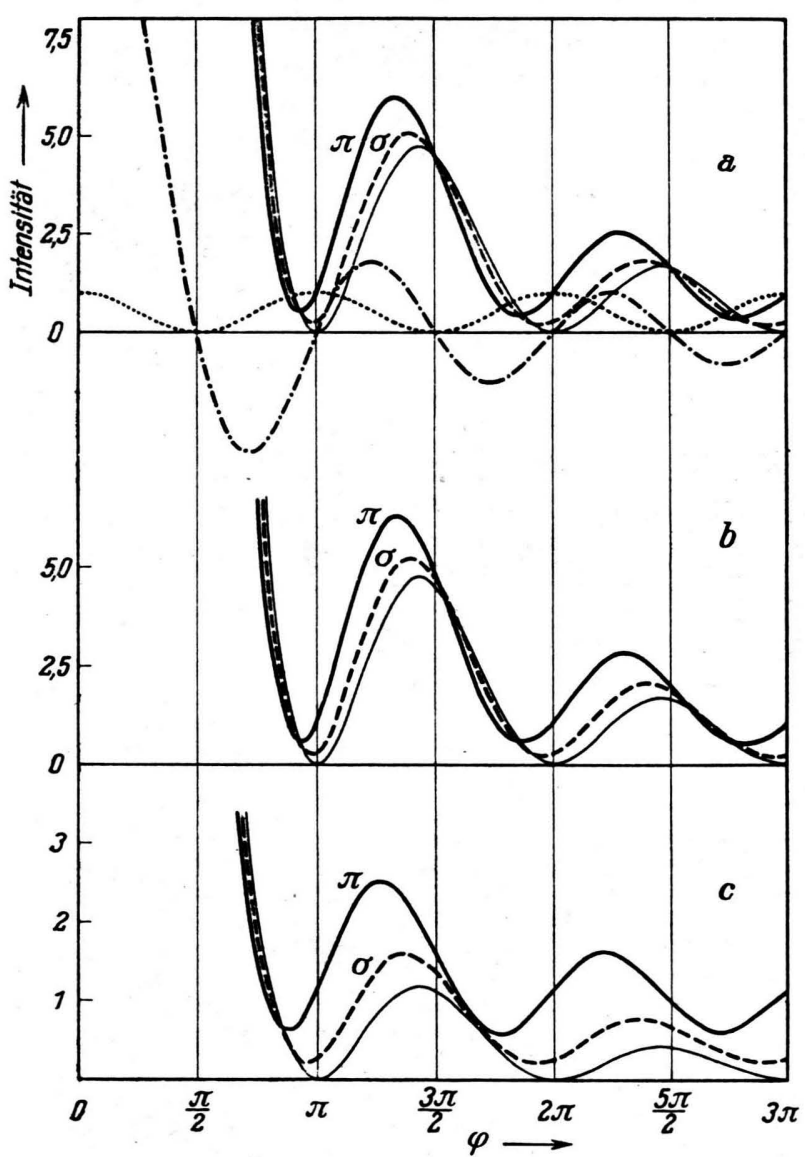

Abb. 8 Theoretischer Intensitätsverlauf.

Komponente, - - - $\sigma$-Komponente, Kirchhoffscher Intensitätsverlauf, ……..... „Doppelspaltinterferenzen" für $\pi$-Komponente, - - - , gemischtes Glied“ für $\pi$-Komponente. a) Intensität nach Gl. (2) mit $a=10 ; b c_{\pi}=1 / 2 ; \delta_{\pi}=45^{\circ} ; b c_{\sigma}=1 / 4 ; \delta_{\sigma}=60^{\circ}$.

Intensität nach Gl. (4) mit zusätzlich $d=1 / 4$; $\delta_{0 \pi}=45^{\circ}$ und $\delta_{0 \sigma}=60^{\circ}$ für b) $a=10$, c) $=5$.

Die Intensität setzt sich also aus drei Teilen zusammen, wovon der erste Teil die Kirchhoffsche Intensitätsverteilung darstellt; der zweite Ausdruck beschreibt die „Doppelspaltinterferenzen“ der beiden von den Spaltbacken ausgehenden Zylinderwellen allein, während der dritte Aus- druck durch das Zusammenwirken beider Anteile zustande kommt (,gemischtes Glied“).

Je nach der Stellung von Polarisator und Analysator zueinander, gibt es eine Reihe von ausgezeichneten Spezialfällen, die in Tab. 1 verzeichnet sind. Abb. 8a zeigt den nach (2) sich ergebenden Intensitätsverlauf für die $\pi$ - und $\sigma$ Komponente für den Spezialfall:

$$
\begin{array}{rlrl}
a=10 ; & b c_{\pi}=1 / 2 ; & \delta_{\pi}=45^{\circ}, \\
b c_{\sigma}=1 / 4 ; & \delta_{\sigma}=60^{\circ} .
\end{array}
$$

Kennzeichnend für den Intensitätsverlauf (2) sind folgende Eigenschaften:

1. An den Stellen $\varphi=\pi / 2,3 \pi / 2,5 \pi / 2$ usw. ist die Intensität gleich der Kirchhoffschen, d.h. die Intensitätsverteilungen der $\pi$ - und $\sigma$-Komponente schneiden sich in diesen Punkten.

2. Bei einem Polarisationsazimut von $45^{\circ}$ und gekreuzten Polarisatoren (Beobachtung I B 1) ergibt sich eine reine $\cos ^{2}$-Verteilung (konstante Amplitude; die Minima sind Nullstellen).

Wie aus den Photometerkurven der Abb. 6 hervorgeht, wird für hinreichend weite Spalte die erste Eigenschaft angenähert bestätigt, wenngleich sich zeigt, daß die Abstände zweier aufeinanderfolgender Schnittpunkte nicht konstant sind und mit wachsender Ordnungszahl größer zu werden scheinen, was um so ausgeprägter in Erscheinung tritt, je enger der Spalt wird, im Widerspruch zur Intensitätsverteilung (2) (vgl. Abb. 8a). Die zweite Eigenschaft ist überhaupt nicht erfüllt (vgl. Abb.4). Die Minima der „Doppelspaltinterferenzen" sind keine Nullstellen. Bemerkenswert ist, daß die Intensität in den Minima um so größer zu sein scheint, je größer der Krümmungsradius der Spaltbacken ist. Bei Spalt II ist sie beträchtlich größer als beim Rasierklingenspalt. Außerdem wächst sie mit zunehmender Ordnungszahl. Die wesentlich größere Intensität der bei gekreuzten Polarisatoren auftretenden ,Doppelspaltinterferenzen" beim Spalt II, verglichen mit Spalt I (vgl. die Belichtungszeiten der Abb. 3), 


\begin{tabular}{|c|c|c|c|}
\hline $\begin{array}{cc}\text { Stellung der Polarisatoren } \\
\beta & \gamma\end{array}$ & $L_{1}$ & $L_{2}$ & $L_{3}$ \\
\hline 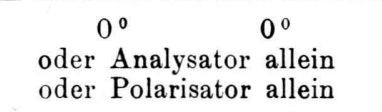 & 1 & $c_{\pi}^{2}$ & $c_{\pi} \cos \delta_{\pi}$ \\
\hline $\begin{array}{c}90^{\circ} \\
\text { oder Analysator allein } \\
\text { oder Polarisator allein }\end{array}$ & 1 & $c_{\sigma}^{2}$ & $c_{\sigma} \cos \delta_{\sigma}$ \\
\hline $\begin{array}{l}\text { unpolarisiert } \\
\text { oder Analysator unter } \mathbf{4 5}^{\circ} \\
\text { oder Polarisator unter } \mathbf{4 5}{ }^{\circ}\end{array}$ & 1 & $\frac{1}{2}\left[c_{\pi}^{2}+c_{\sigma}^{2}\right]$ & $\frac{1}{2}\left[c_{\pi} \cos \delta_{\pi}+c_{\sigma} \cos \delta_{\sigma}\right]$ \\
\hline $45^{\circ}$ & 1 & $\frac{1}{4}\left[c_{\pi}^{2}+c_{\sigma}^{2}+2 c_{\pi} c_{\sigma} \cos \left(\delta_{\pi}-\delta_{\sigma}\right)\right]$ & $\frac{1}{2}\left[c_{\pi} \cos \delta_{\pi}+c_{\sigma} \cos \delta_{\sigma}\right]$ \\
\hline $45^{\circ}$ & 0 & $\frac{1}{4}\left[c_{\pi}^{2}+c_{\sigma}^{2}-2 c_{\pi} c_{\sigma} \cos \left(\delta_{\pi}-\delta_{\sigma)}\right]\right.$ & 0 \\
\hline
\end{tabular}

Tab. 1. Werte der Koeffizienten $L$ für ausgezeichnete Stellungen der Polarisatoren.

läßt sich dadurch verstehen, daß beim Spalt II mit der kleineren Krümmung die "reflektierende" Zone b größer ist als beim Rasierklingenspalt (vgl. Abb. 2).

Anna hme 2

Das beobachtete Fehlen von Schnittpunkten der Intensitätskurven für die $\pi^{-}$und $\sigma$-Komponente bei engen Spalten sowie die in den Minima der „Doppelspaltinterferenzen“ beobachtete endliche Intensität machen eine Abänderung der Annahme 1 notwendig. Eine Erklärung für diese Tatsachen wird ermöglicht durch die Vorstellung, daß die Amplitude der von einer Spaltbacke emittierten Randwelle für die verschiedenen Richtungen verschieden groß ist. Eine solche Annahme scheint auch physikalisch plausibel zu sein. Denn die Annahme von richtungsunabhängigen Zylinderwellen entspricht zweifellos nicht den Tatsachen. So wird z. B. (vgl. Abb. 7, Kurve b) die Zone b der linken Spaltbacke in einer Richtung, die von der Richtung der einfallenden Welle um den Winkel $\propto$ nach links abweicht, weniger Licht emittieren als in die um denselben Winkel nach rechts abweichende Richtung. Das Strahlungs- diagramm der Zone $b$ wird wesentlich von der geometrischen Gestalt der Spaltbacken abhängig sein. Als eine erste Näherung nehmen wir an, das Strahlungsdiagramm sei eine Cardioide $[1 \pm(d / a) \varphi]$, d. h. wir verbessern den Ansatz (1) der Annahme 1 durch den neuen Ansatz $b c \exp (i \delta)[1-(d / a) \varphi] \exp (i \delta)$ für die rechte bzw. $b c \exp (i \delta)[1+(d / a) \varphi] \exp (-i \delta)$ für die linke Randwelle. Dabei ist $d$ eine Konstante, deren Größe im wesentlichen vom Krümmungsradius der Spaltbacken abhängen wird. Es ist ohne weiteres einleuchtend, daß mit dieser Vorstellung die Beobachtung verständlich wird, daß die Minima der bei gekreuzten Polarisatoren auftretenden „Doppelspaltinterferenzen“ keine Nullstellen sind, da nunmehr die beiden Randwellen in einer bestimmten Richtung $\alpha$ nicht mehr gleiche Intensität besitzen. Ferner fällt jetzt in den Raum außerhalb der Tangente $\mathrm{T}$ (Abb. 7) bei gekreuzten Polarisatoren nur noch Licht von der gegenüberliegenden Spaltbacke. Es können also dort - in Übereinstimmung mit der Beobachtung - keine „Doppelspaltin̄terferenzen" mehr auftreten. Mit diesem Ansatz ergibt sich speziell für die Intensitätsverteilung der $\pi$-Komponente der Ausdruck

$$
I_{\pi}=a^{2} \frac{\sin ^{2} \varphi}{\varphi^{2}}+4 b^{2} c_{\pi}^{2}\left\{\cos ^{2} \varphi+\frac{d^{2}}{a^{2}} \varphi^{2} \sin ^{2} \varphi\right\}+4 b c_{\pi}\left\{a \frac{\sin \varphi \cos \varphi}{\varphi} \cos \delta_{\pi}+d \sin ^{2} \varphi \sin \delta_{\pi}\right\} .
$$

Die der $\sigma$-Komponente entsprechende Formel ergibt sich, wenn $c_{\pi}$ durch $c_{\sigma}$ und $\delta_{\pi}$ durch $\delta_{\sigma}$ ersetzt werden.

Der Ausdruck (3) besteht abermals aus drei
Teilen, wovon der erste wiederum die Kirchhoffsche Intensitätsverteilung bedeutet. Der zweite Ausdruck beschreibt wiederum die „Doppelspaltinterferenzen" der beiden Randwellen allein, wäh- 
Abb. 3

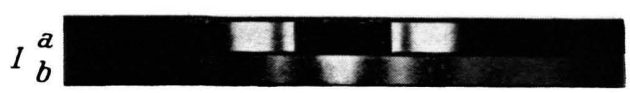

II $\begin{aligned} & a \\ & b\end{aligned}$

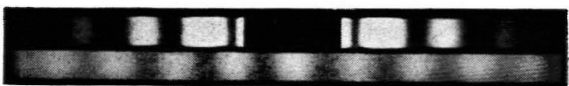

Abb. $4:$

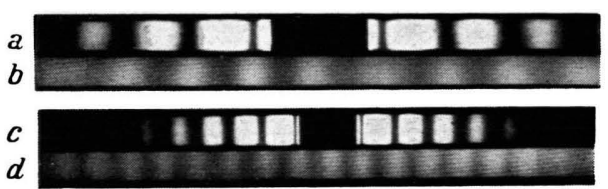

Abb. 3. Spalt I / I a) Beugungsaufnahmen bei unpolarisiertem Licht, Belichtungszeit 30 Sek.

Höhe $8 \mathrm{~mm}$ ( I b) ,Doppelspaltinterferenzen“ bei gekreuzten Polarisatoren, Belichtungszeit 3,5 Stdn.

Spalt II (II a) Beugungsaufnahmen bei unpolarisiertem Licht, Belichtungszeit 20 Sek.

Höhe $20 \mathrm{~mm}$ (II b) „Doppelspaltinterferenzen“ bei gekreuzten Polarisatoren, Belichtungszeit 2,5 Min. Spaltbreite in beiden Fällen etwa $21 \mu$; Breite des Beleuchtungsspaltes $800 \mu$; Lichtquelle HBO 501 mit Schott-Filter OG 4. (Die störenden horizontalen Streifen stammen von Interferenzen an der fehlerhaften Kittung der Glan-Thompson-Prismen.)

Abb. 4. Spalt II | Beugungsaufnahmen bei unpolarisiertem Licht, Spaltbreite und Belichtungszeiten: Höhe $20 \mathrm{~mm}\}$ a) etwa $21 \mu, 4$ Min.; b) etwa $21 \mu$, 30 Min.; c) etwa $46 \mu, 4$ Min.; d) etwa $46 \mu, 20 \mathrm{Min}$. Breite, des Beleuchtungsspaltes $200 \mu$; Lichtquelle HBO 501 mit Wrattenfilter.

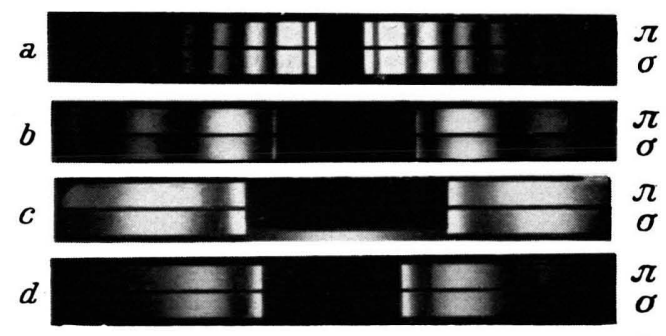

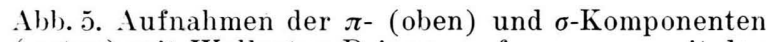
(unten) mit Wollaston-Prisma; aufgenommen mit den D-Linien.

Spaltbreite

Beetwa

Spalt II ( a) 44 !

Höhe $20 \mathrm{~mm}\{\mathrm{~b}) \quad 18 \%$

11,11

Spalt I

Höhe $6,7 \mathrm{~mm}$ c)

d) leuchtungs-

spalt

- $100 \mu$

$200 \mu$

$200 " 1$

$200 \mu$
Be-

lichtungszeit

3 Stdn.

5 Stdn.

14 Stdn.

16 Stdn.

\section{H.Schüler und L.Reinebeck, Über ein neues OD-Spektrum im Sichtbaren (S.560)}

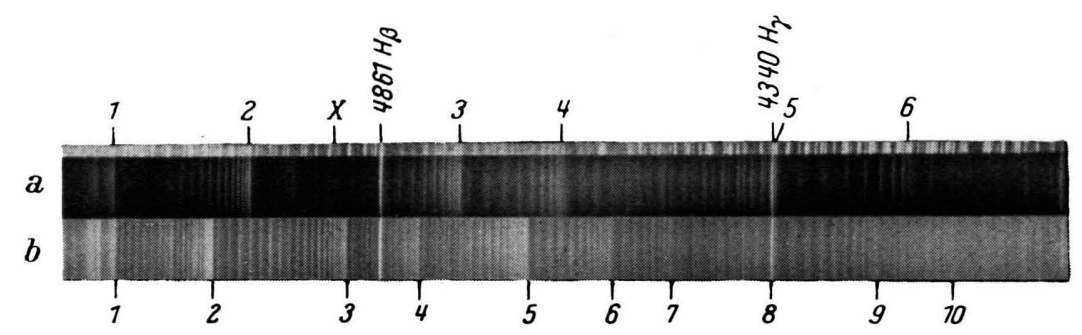

Abb. 1. a: Emissionsspektrum von $\mathrm{OH}$ im Sichtbaren (l. c.), b: Emissionsspektrum von OD im Sichtbaren. Die Bandenkanten haben die gleiche Numerierung wie im Text. Eisen-Vergleich. (Aufnahme mit dem Drei-Prismenspektrographen nach Zeiß-Försterling, $\mathrm{f}=27 \mathrm{~cm}$.)

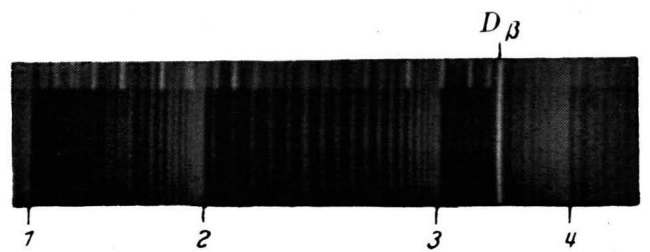

Abb.3. Ausschnitt aus dem OD-Spektrum. Einzelheiten der Rotationsstruktur der Banden 2 und 3 mit EisenVergleich. (Aufnahmen mit dem Drei-Prismenspektrographen nach Zeiß-Försterling, $\mathrm{f}=85 \mathrm{~cm}$.) 


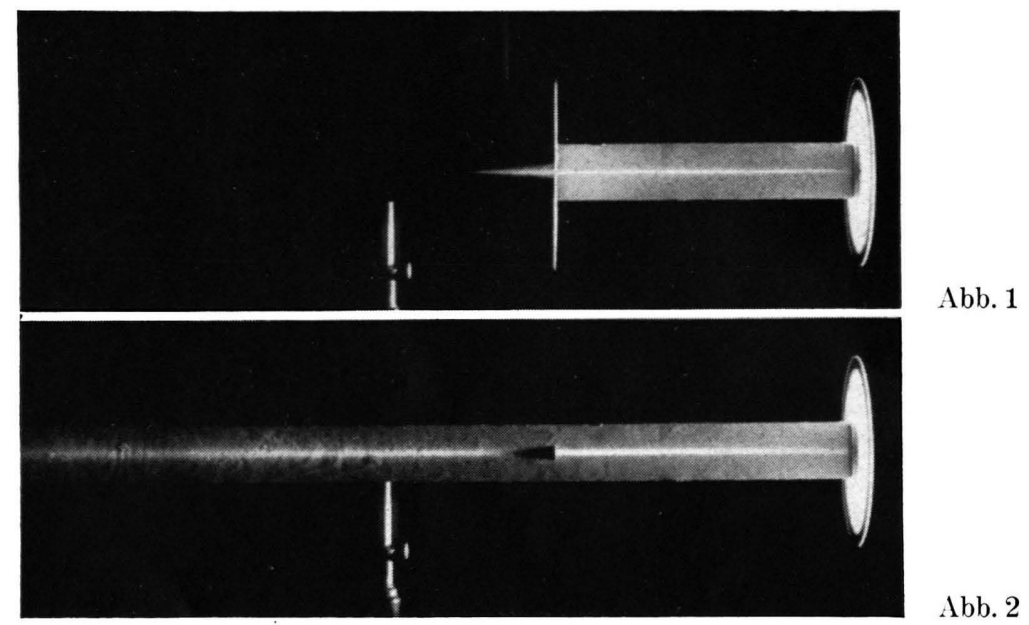

Abb. 1. Die leuchtende Brennachse durchsetzt eine Blende und erlischt dann. Abb. 2. Sie taucht hinter einem schattenwerfenden Hindernis wieder auf.

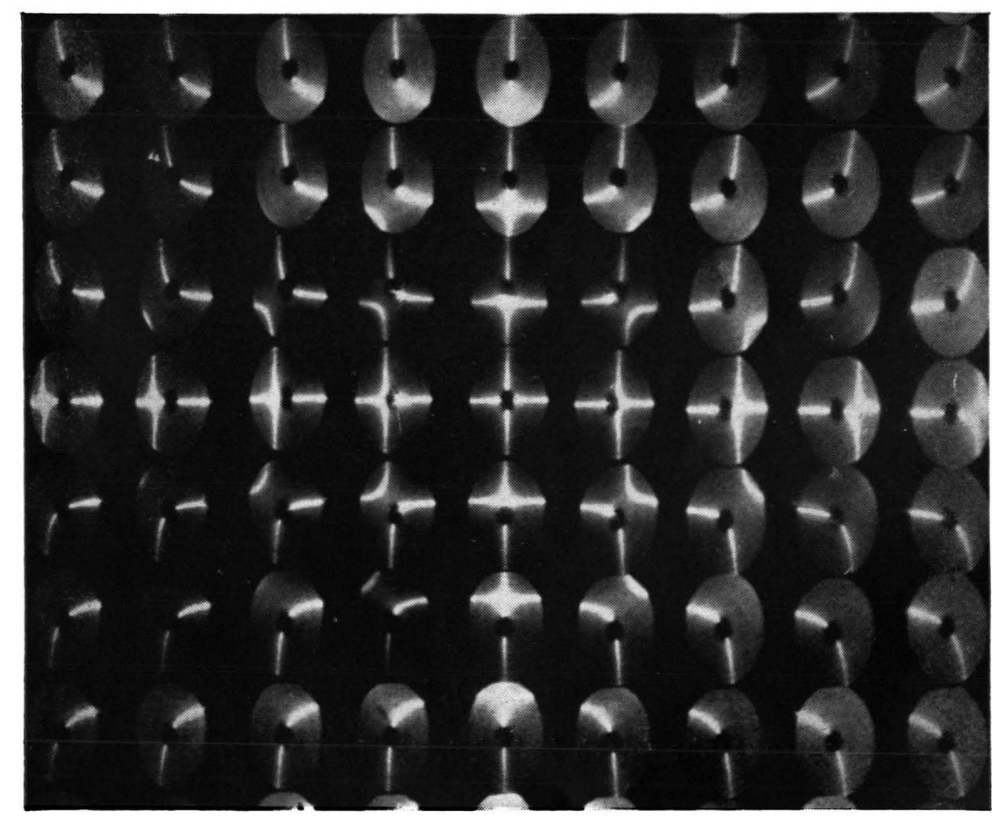

Abb. 5. Netz der mitarbeitenden Bereiche einer Kreisringplatte. 
rend der dritte das ,gemischte Glied“ darstellt. Die Diskussion der Intensitätsformel (3) ergibt folgendes: Die Punkte gleicher Intensität von $\pi^{-}$ und $\sigma$-Komponente Fiegen nicht mehr an den Stellen $\varphi=\pi / 2,3 \pi / 2,5 \pi / 2$ usw. (vgl. Abb. 8 b). Sie rücken in der Tat, entsprechend den Beobachtungen, mit wachsender Ordnungszahl zu immer größeren Werten von $\varphi$. Es ist auch durch geeignete Wahl des Parameters $d$ des Strahlungsdiagramms möglich, zu erreichen, daß die Intensitätskurven für $\pi$ und $\sigma$ sich nicht mehr schneiden. Doch folgt aus (3), im Gegensatz zu den Beobachtungen, daß die Stelle, wo zum erstenmal $\pi^{-}$ und $\sigma$-Komponente sich nicht mehr schneiden, unabhängig von der Spaltbreite bei demselben $\mathrm{Ab}$ lenkungswinkel $\propto$ liegen müßte. Die Photometerkurven der Abb. 6 zeigen aber, daß diese Stelle (außer von der Konstanten $d$ ) von der Spaltbreite abhängig ist und bei um so kleineren Ablenkungswinkeln liegt, je enger der Spalt ist.

Die bei gekreuzten Polarisatoren zu erwartenden „Doppelspaltinterferenzen“ besitzen nach (3), entsprechend der Beobachtung, keine Nullstellen mehr. Die Intensität in den Minima nimmt ebenfalls im Einklang mit den Beobachtungen mit wachsendem Ablenkungswinkel zu.

Annahme 3

DieTatsache, daß das Verschwinden der Schnittpunkte für die Intensitätskurven der $\pi^{-}$und $\sigma^{-}$
Komponente von der Spaltbreite abhängig ist, im Gegensatz zu der aus der Annahme 2 folgenden Intensitätsformel (3), legt es nahe, die Amplitude der von einer Spaltbacke emittierten Randwelle als abhängig von der Spaltbreite anzusetzen. Ein solcher Ansatz läßt sich physikalisch rechtfertigen durch den Umstand, daß die Spaltbacken ja nicht nur durch die einfallende Welle beleuchtet werden, sondern auch mit den von ihnen ausgehenden Randwellen sich gegenseitig Licht zustrahlen. Berücksichtigen wir in erster Näherung nur das einmal "gespiegelte“ Licht, so wird die Amplitude der einen Spaltbacke durch Zustrahlung von der anderen um den Betrag $b c / a$ vergrößert sein. Dieser Anteil wird freilich in einem anderen Phasenzustand sich befinden als derjenige, der von der primär einfallenden Welle verursacht ist. Diesem Umstand wird durch Einführung einer weiteren Phasenkonstanten $\delta_{0}$ Rechnung getragen. Damit ergibt sich in Erweiterung der Annahme 2 für die von der rechten Spaltbacke ausgehende Randwelle der Ansatz $b c \exp (i \delta)$ $\cdot\left[1+\frac{b c}{a} \exp \left(i \delta_{0}\right)\right]\left(1-\frac{d}{a} \varphi\right) \exp (i \varphi)$ und für die linke Spaltbacke $b c \exp (i \delta)\left[1+\frac{b c}{a} \exp \left(i \delta_{0}\right)\right]$ $\cdot\left(1+\frac{d}{a} \varphi\right) \exp (i \varphi)$.

Für die Intensitätsverteilung folgt dann (wiederum für die $\pi$-Komponente)

$$
\begin{aligned}
& I_{\pi}=a^{2} \frac{\sin ^{2} \varphi}{\varphi^{2}}+4 b^{2} c_{\pi}^{2}\left(1+2 \frac{b c_{\pi}}{a} \cos \delta_{0 \pi}\right)\left\{\cos ^{2} \varphi+\frac{d^{2}}{a^{2}} \varphi^{2} \sin ^{2} \varphi\right\} \\
& +4 b c_{\pi}\left\{a \frac{\sin \varphi \cos \varphi}{\varphi}\left[\cos \delta_{\pi}+\frac{b c_{\pi}}{a} \cos \left(\delta_{0 \pi}+\delta_{\pi}\right)\right]+d \sin ^{2} \varphi\left[\sin \delta_{\pi}+\frac{b c_{\pi}}{a} \sin \left(\delta_{0 \pi}+\delta_{\pi}\right)\right]\right\} .
\end{aligned}
$$

Da der „Reflexionskoeffizient“ $c$ von 1 nicht sehr verschieden sein wird, macht sich die gegenseitige Zustrahlung der Spaltbacken erst bemerkbar, wenn die Spaltbreite $a$ vergleichbar mit der Randzone $b$ wird. Beim Spalt II kann diese Zone auf einige wenige $\mu$ (etwa $3 \mu$ ) geschätzt werden. Wie Abb. 6 zeigt, verschwinden die Schnittpunkte der $\pi^{-}$und $\sigma$-Kurven bei einer Spaltbreite von etwa $18 \mu$, d. h. für $b / a$ ungefähr $1 / 6$.

Wir haben den aus (4) folgenden Intensitätsverlauf in den Abb. $8 \mathrm{~b}$ und $\mathrm{c}$ aufgezeichnet für zwei verschiedene Spaltbreiten $a=10$ (Teilbild b) und $a=5$ (Teilbild c). Für die anderen Konstanten der Gl. (4) wurden folgende Werte gewählt:

$$
\begin{array}{rlll}
d=1 / 4 ; & b c_{\pi}=1 / 2 ; & \delta_{\pi}=45^{0} ; & \delta_{0 \pi}=45^{0}, \\
b c_{\sigma}=1 / 4 ; & \delta_{\sigma}=60^{\circ} ; & \delta_{0 \sigma}=60^{\circ} .
\end{array}
$$

Dies würde für $c_{\pi}=0,5$ und $c_{\sigma}=0,25$ beì $b=3 \mu$ Spaltbreiten von $30 \mu$ bei Teilbild b bzw. $15 \mu$ bei Teilbild c ergeben. Während für $a=10$ die $\pi$ - und $\sigma$-Kurven sich noch schneiden, ist dies für $a=5$ nicht mehr der Fall.

Es kann freilich nicht behauptet werden, daß die den Abb. 8 b und c zugrunde gelegten Konstanten für den untersuchten Spalt II zutreffend sind. Insbesondere ist es fraglich, ob die als richtungsunabhängig angenommene Phasenverschiebung $\delta$ 
der Wirklichkeit entspricht. Es sollte lediglich gezeigt werden, daß mit der aus der Annahme 3 folgenden Intensitätsformel (4) Kurven erhalten werden können, welche die wesentlichsten Eigenschaften der beobachteten Intensitätsverteilung aufweisen.

Zum Schluß sei noch hervorgehoben, daß die von uns eingeführte "physikalische Randwelle“ nicht identisch ist mit der in der Sommerfeldschen
Beugungstheorie auftretenden ,mathematischen Randwelle“. Bekanntlich kann die Kirchhoffsche und auch die.Sommerfeldsche Lösung aufgefaßt werden als Summe aus der einfallenden Welle und einer ,mathematischen“ Randwelle. Von diesem Standpunkt aus gesehen ist nach unserer Ansicht die. Annahme einer weiteren ,physikalischen" Randwelle erforderlich, um die beobachteten Erscheinungen zu verstehen.

\title{
Didaktisches zur Lichtbeugung
}

\author{
Von Walther Kossel \\ Aus dem Physikalischen Institut der Universität Tübingen \\ (Z. Naturforschg. 4 a, 506-509 [1949]; eingegangen am 19. März 1949)
}

Didaktische Hinweise zu einer kürzlich hier beschriebenen Beugungsdemonstration.

Z um 80. Geburtstag Sommerfelds ${ }^{1}$ wurde hier ein Versuch beschrieben, der erlaubt, Grundsätzliches zur Lichtbeugung zu diskutieren. Da die Erscheinungen außerdem hell genug sind, in einem ganzen Hörsaal gesehen $\mathrm{zu}$ werden, geben wir hier einige Hinweise auf Didaktisches ${ }^{2}$.

Das beugende System ist eine Glasplatte, in die konzentrische Ringe geritzt sind, deren Radien sich statistisch verteilen. Sie wurde auf der Drehbank mit Schmirgelpapier hergestellt. Inzwischen hat sich gezeigt, daß man die Schärfe der Erscheinungen merklich bessert, wenn man statt des Schmirgelpapiers, dessen Körner leicht zur Seite gedrückt oder mit fortgerissen und herumgewälzt werden, so daß einige Ringspuren nicht sauber konzentrisch ausfallen, Schmirgelstein selbst verwendet.

1. Liegt die Quelle auf der Achse, d. h. steht die Platte senkrecht zur primären Einfallsrichtung, so zeigt sich hinter der Platte ein längs der Achse führendes Interferenzmaximum, das wir kurz die „Brennachse“ nannten. Da es aussieht wie ein längs der Achse führender Strahl, wirkt überraschend, da\& die helle Linie kurz hinter einer offenen Blende erlischt und hinter einem kleinen Hindernis bald wieder auftaucht. Wir zeigen diese Erscheinungen in Abb. $1^{*}$ und 2*. Man erkennt an der Form des Schattenwurfs gut die Herkunft des

1 W. K o s s e 1, Z. Naturforschg. 3a, 496 [1948].

2 Die Bilder, die wir dabei zeigen, sind zum größeren Teil von Hrn. cand. phys. F. S a u r hergestellt worden.

* S. Tafel, S. 504 b.
Phänomens aus dem gesamten von Ringen erfüllten Bereich der Beugungsplatte. Eine quantitative Behandlung der Intensitätsverteilung auf und um die Brennachse gibt die hier folgende Arbeit von W. B r a u nek.

2. Didaktisch sehr brauchbar ist das Phänomen zur Behandlung der Extremalprinzipien. Sie wollen den tatsächlichen Ablauf eines Geschehens als einen extremalen unter den möglichen auszeichnen. Das Unbehagliche an ihnen, das man insbesondere an den mechanischen Sätzen dieser Art verspürt, rührt daher, daß man von vornherein irgendeine Gesamtheit als „möglich“ kennzeichnen muß. Darin liegt eine Willkür. Die Wahl dieser Gesamtheit ergibt aber die verschiedenen Formen der mechanischen Prinzipien dieser Art. Einmal werden Abläufe gleicher Zeitdauer. ein anderes Mal solche gleicher Energie usw. als möglich vorausgesetzt, und es wird gezeigt, daß unter ihnen der wirkliche Ablauf eine Extremaleigenschaft besitzt. Darin, was so als möglich erklärt wird, besteht Willkür.

Das Fermatsche Prinzip der kürzesten Lichtzeit zeichnet sich unter den Sätzen dieser Art als besonders durchsichtig aus. Wenn z. B. die geradlinige Ausbreitung von einer freistehenden Quelle zu einem freistehenden Aufpunkt als die Wahl des Weges kürzester Lichtzeit erklärt wird, richtet man die Aufmerksamkeit darauf, daß das Licht im freien Raum auch andere offene Wege von der Quelle zum Aufpunkt vorfindet. Alle diese Wege werden also zunächst als ,möglich“ vorausgesetzt. In der Tat leuchtet ja die Quelle nach allen Seiten, 